ANNALES

POLONICI MATHEMATICI

55 (1991)

\title{
Univalent functions with logarithmic restrictions
}

\author{
by A. Z. Grinshran (Leningrad)
}

\begin{abstract}
It is known that univalence property of regular functions is better understood in terms of some restrictions of logarithmic type. Such restrictions are connected with natural stratifications of the studied classes of univalent functions. The stratification of the basic class $\mathcal{S}$ of functions regular and univalent in the unit disk by the Grunsky operator norm as well as the more general one of the class $\mathfrak{M}^{*}$ of pairs of univalent functions without common values by the $\tau$-norm (this concept is introduced here) are given in the paper. Moreover, some properties of univalent functions whose range has finite logarithmic area are considered. To apply the logarithmic restrictions a special exponentiation technique is used.
\end{abstract}

Introduction. The theory of univalent functions as well as the whole geometric function theory is characterized by the necessity of joint consideration of both analytical and geometric aspects. If one is concerned with simply connected domains, as in the present paper, then the classical Riemann mapping theorem is of fundamental importance. In particular, it establishes a direct correspondence between the class $\mathfrak{A}$ of regular and univalent functions $f(z)=c_{1} z+\ldots$ in the unit disk $E=\{z:|z|<1\}$ and the set of simply connected domains in the finite complex plane $\mathbb{C}$ containing the origin. It is known that the univalence property of regular functions is better understood in terms of specific restrictions of logarithmic type (or equivalent to them), stemming from geometric ideas (see e.g. $[12 ; 3 ; 8]$ ). One means here restrictions on compositions of the form $\log \circ \Phi$ where $\Phi$ is some operator on the studied class of univalent functions (e.g. $\mathfrak{A}$ ) such that $\Phi(f)(f \in \mathfrak{A})$ is a regular function of one or two complex variables which does not vanish in the corresponding domain. Those logarithimic restrictions are connected with natural stratifications of the set of simply connected domains and of the corresponding class of univalent functions,

1991 Mathematics Subject Classification: 30C50, 30C75, 30C60.

Key words and phrases: univalent functions, logarithmic restrictions, Grunsky operator, quasiconformal extension, exponentiation technique, functions without common values, homeomorphic assembling. 
and thus with geometric and analytical properties of the studied functions. Analysis of interdependence and joint application of those properties are usually rather difficult. To overcome those difficulties when solving various problems the logarithmic restrictions and special exponentiation technique are used.

$\S 1$. Functions whose range has a finite logarithmic area. Investigating some known subclasses of the class $\mathfrak{A}$ :

- bounded functions,

- functions with finite range area,

— Bieberbach-Eilenberg functions (see §3) and others,

led to the consideration of a more convenient family of functions defined by a restriction of logarithmic type. On the one hand, this family contains the above-mentioned subclasses and retains a number of their properties, and on the other hand it seems more natural from the point of view of joint consideration of geometric and analytical properties of functions. One means here those functions in the class $\mathfrak{A}$ whose range has finite logarithmic area. The corresponding class $\mathcal{A}_{\mathcal{S}}$ of functions with a suitable normalization was introduced in [9]. The stratification of both the class $\mathfrak{A}$ of univalent functions and the set of simply connected domains according to the value of the logarithmic area of the image domain has some advantages over the traditional approaches.

For any function $g(z)$ regular in $E$, denote by $\sigma(g)$ the area of its image domain $g(E)$ in the corresponding Riemann surface, divided by $\pi$.

Also, denote by $\sigma(B)$ the area of a measurable plane set $B$ divided by $\pi$. The logarithmic area of any simply connected domain $B=f(E)$, containing zero, where $f \in \mathfrak{A}$, is defined to be $\pi \sigma(\log (f(z) / z))$ [9]. For such domains and Riemann functions mapping onto them the value of A-measure [4] is of paramount importance. $\mathbf{A}(B)$ (or $\mathbf{A}(f))$ is defined by

$$
\mathbf{A}(B)=2 \log R+\sigma(\log (f(z) / z))
$$

where $R$ is the inner radius of the domain $B$ with respect to zero. A number of non-trivial properties of $\mathbf{A}$-measure were established in [4]. In particular, we proved the sharp inequality $\mathbf{A}(B) \leq \log \sigma(B)$ and established that the $\mathbf{A}$ measure (unlike the logarithmic area) is monotonic as a function of domain. We also recall that for simply connected domains the $\mathbf{A}$-measure coincides (see $[4 ; 9]$ ) with the Teichmüller reduced logarithmic area [15].

An example of a function in $\mathfrak{A}$ with the infinite logarithmic area of its range is the Koebe function $\mathbf{k}_{1}(z)=z(1-z)^{-2}$. Any bounded function in $\mathfrak{A}$ or even any function in $\mathfrak{A}$ with finite range area [4; 9 gives an opposite example. The function $\widetilde{f}(z)=-\log (1-z) \in \mathfrak{A}$ is the simplest example of 
an unbounded function whose image domain has an infinite area but a finite logarithmic area.

In fact, $\partial \widetilde{f}(E)$ has a parametric representation

$$
x=-\log 2-\log \sin \theta / 2, \quad y=(\pi-\theta) / 2 ; \quad 0<\theta<2 \pi,
$$

so $\widetilde{f}(E)$ is a domain contained in the half-strip

$$
\{z:|\operatorname{Im} z|<\pi / 2, \operatorname{Re} z>-\log 2\} .
$$

Therefore its logarithmic area, equal to $\pi \mathbf{A}(\tilde{f})$, does not exceed (see [4])

$$
\pi\left(\log \left[1+(\pi / 2)^{2}\right]+I\right) \quad \text { where } \quad I=2 \int_{1}^{\infty} d x \int_{0}^{\pi / 2} d y /\left(x^{2}+y^{2}\right)<\pi .
$$

In the same way we can show that for any function $f$ in $\mathfrak{A}$, if the range of $f$ is in some strip, then it has finite logarithmic area. Assume that

$$
f(E) \subset \Pi_{H}=\{z:|\operatorname{Im} z|<H / 2\} .
$$

Since the function

$$
f_{H}(z)=(H / \pi) \log [(1+z) /(1-z)] \in \mathfrak{A}
$$

maps $E$ onto $\Pi_{H}$ and $\mathbf{A}(f) \leq \mathbf{A}\left(f_{H}\right)$ (see [4]) we have

$$
\begin{aligned}
\sigma(\log (f(z) / z)) & \leq-2 \log \left|f^{\prime}(0)\right|+\mathbf{A}\left(f_{H}\right) \\
& <\log \left[\left|H / f^{\prime}(0)\right|^{2}\left((4 / \pi)^{2}+1\right)\right]+\pi .
\end{aligned}
$$

According to the definition in [9] the class $\mathcal{A}_{\mathcal{S}}$ consists of those functions $f(z)=c_{1} z+\ldots \in \mathfrak{A}$ for which $\mathbf{A}(f) \leq 0$. It follows from the definition that this class only contains functions whose range has finite logarithmic area. On the other hand, any $g \in \mathfrak{A}$ whose range has finite logarithmic area can be represented as $g(z)=\xi f(z)$ where $f \in \mathcal{A}_{\mathcal{S}}$, and $\xi$ is a constant.

In [9] a number of sharp inequalities for functions $f(z)=c_{1} z+\ldots \in \mathcal{A}_{\mathcal{S}}$ were established, in particular, the following ones:

$$
\begin{gathered}
\frac{1}{2 \pi} \int_{|z|=1}|f(z)||d z| \leq\left|c_{1}\right|^{1 / 2}, \\
\frac{1}{2 \pi} \int_{|z|=1}|f(z)|^{2}|d z| \leq 1, \\
\frac{1}{\pi} \iint_{E}|f(z) / z|^{4} d \sigma \leq 1, \\
|f(z)| \leq|z|\left(1-|z|^{2}\right)^{-1 / 2}, \quad z \in E .
\end{gathered}
$$

Equality in (2)-(5) occurs only for functions of the form

$$
f(z)=c_{1} z(1-\zeta z)^{-\varepsilon}, \quad\left|c_{1}\right|=\left(1-|\zeta|^{2}\right)^{\varepsilon^{2} / 2}, \quad \zeta \in E,
$$


for $\varepsilon=1$ in (3)-(5) and for $\varepsilon=2$ in (2).

The inequalities (3) and (5) are extensions to the whole class $\mathcal{A}_{\mathcal{S}}$ of the known Lebedev and Jenkins inequalities respectively for the BieberbachEilenberg functions. The inequalities (2) and (4) were obtained in [9] for the class $\mathcal{A}_{\mathcal{S}}$.

Consider the problem of estimating the Taylor coefficients of functions in the class $\mathcal{A}_{\mathcal{S}}$. For functions

$$
f(z)=c_{1} z+\ldots \in \mathfrak{A}
$$

whose image domain area does not exceed $\pi$ (in particular, for functions bounded in $E$, with $|f(z)|<1$ ) the inequality $\sigma(f)=\sum_{n=1}^{\infty} n\left|c_{n}\right|^{2} \leq 1$ certainly holds. Hence we have

$$
\left|c_{n}\right| \leq n^{-1 / 2} \quad(n=1,2, \ldots) .
$$

For Bieberbach-Eilenberg functions in $\mathfrak{A}$ the inequality (7) (and even a stronger one) also holds and it cannot be improved in the sense of the growth order in $n$ as $n \rightarrow \infty$. The result for Bieberbach-Eilenberg functions was first published independently by Z. Nehari and D. Aharonov in 1970. The approach used by them as well as by the author [4] allows one to extend (7) to the whole class $\mathcal{A}_{\mathcal{S}}$. To do this, we need an exponential LebedevMilin inequality. Let us formulate it as a lemma, together with another Lebedev-Milin exponential inequality used later in the paper.

Lemma 1 (see e.g. [12, Ch. 2]). For any sequence of complex numbers $\left\{A_{n}\right\}_{n=1}^{\infty}$, let the coefficients $D_{n}$ be defined by the expansion $\sum_{n=0}^{\infty} D_{n} z^{n}=$ $\exp \left\{\sum_{n=1}^{\infty} A_{n} z^{n}\right\}$. Then

$$
\begin{gathered}
\left|D_{n}\right| \leq \exp \left\{\frac{1}{2} \sum_{l=1}^{n}\left(l\left|A_{l}\right|^{2}-1 / l\right)\right\} \quad(n=1,2, \ldots), \\
\sum_{n=0}^{\infty}\left|D_{n}\right|^{2} \leq \exp \left\{\sum_{n=1}^{\infty} n\left|A_{n}\right|^{2}\right\} .
\end{gathered}
$$

TheOREM 1. Let $f(z)=c_{1} z+\ldots \in \mathcal{A}_{\mathcal{S}}$. Then the inequality (7) holds, and it is sharp in the sense of the growth order in $n$ as $n \rightarrow \infty$. For $n=1$ equality in (7) occurs if and only if $f(z)=c_{1} z,\left|c_{1}\right|=1$. For $n=2$ the stronger inequality $\left|c_{2}\right| \leq e^{-1 / 2}$ holds, with equality if and only if

$$
f(z)=c_{1} z \exp \{\kappa z-1 / 2\}, \quad\left|c_{1}\right|=|\kappa|=1 .
$$

Proof. For $n=1$ the assertion immediately follows from the definition of $\mathcal{A}_{\mathcal{S}}$ [9]. To obtain the estimate for $\left|c_{2}\right|$, also from the definition of $\mathcal{A}_{\mathcal{S}}$ we have $2 \log \left|c_{1}\right|+\left|c_{2} / c_{1}\right|^{2}+\ldots \leq 0$, and hence $\left|c_{2}\right|^{2} \leq-\left|c_{1}\right|^{2} \log \left|c_{1}\right|^{2} \leq e^{-1}$ as desired. Since the function (8) is in $\mathcal{A}_{\mathcal{S}}$, we also obtain the equality assertion. 
Now let $n \geq 3$. Taking, in Lemma $1, A_{l}=\{\log (f(z) / z)\}_{l}(l=1,2, \ldots)$, by (1) and the inequality $\log n+c<\sum_{l=1}^{n} 1 / l$ where $c$ is the Euler constant, we have from the first inequality of Lemma 1

$$
\left|c_{n}\right|<\left[e^{c}(n-1)\right]^{-1 / 2}<n^{-1 / 2} .
$$

The sequence of functions

$$
f_{n}(z)=z\left[n^{1 / 2}-(n-1)^{1 / 2} z\right]^{-1}=\sum_{l=1}^{\infty} c_{l}^{(n)} z^{l} \in \mathcal{A}_{\mathcal{S}} \quad(n=1,2, \ldots)
$$

shows that (7) cannot be improved in the sense of the growth order in $n$ since

$$
\left|c_{n}^{(n)}\right|=n^{-1 / 2}\left(1-n^{-1}\right)^{(n-1) / 2} \sim(e n)^{-1 / 2} \quad(n \rightarrow \infty) .
$$

The theorem is proved.

Note that when all the mentioned functionals are estimated on $\mathcal{A}_{\mathcal{S}}$ the extremal functions are of the form (6), with the condition $|\varepsilon-1| \leq|\zeta|^{-1}$ ( $\varepsilon$ real) ensuring that those functions are in $\mathcal{A}_{\mathcal{S}}$. This also concerns the function (8) which is obtained from (6) by letting $\varepsilon \rightarrow \infty$.

An interesting conjecture is that for each $n \geq 3$, sup $\left|c_{n}\right|$ over the class $\mathcal{A}_{\mathcal{S}}$ is realized by a function of the form (6) with some $\zeta \in E$ and $0<\varepsilon \leq$ $1+|\zeta|^{-1}$, both depending on $n$.

$\S 2$. Functions with a restriction on the norm of the Grunsky operator. For $f \in \mathfrak{A}$ the expansion

$$
\log \frac{f(z)-f(\zeta)}{z-\zeta}=\sum_{n, l=0}^{\infty} \alpha_{n, l} z^{n} \zeta^{l} \quad(z, \zeta \in E)
$$

generates the Grunsky coefficients $\alpha_{n, l}$ and the Grunsky operator

$$
G_{f}=\left\{\sqrt{n l} \alpha_{n, l}\right\}_{n, l=1}^{\infty}: l^{2} \rightarrow l^{2}
$$

where $l^{2}$ is the Hilbert space of complex sequences with the inner product $\langle x, y\rangle=\sum_{n=1}^{\infty} x_{n} \bar{y}_{n}$ and the norm

$$
\|x\|=\left(\sum_{n=1}^{\infty}\left|x_{n}\right|^{2}\right)^{1 / 2} .
$$

It is known that the necessary and sufficient condition of univalence established by Grunsky is equivalent to the norm $\left\|G_{f}\right\|$ of the Grunsky operator being at most 1 (see e.g. $[3 ; 13]$ ).

Following [7] we denote by $\mathcal{S}(k), k \in[0,1]$, the class of functions $f(z)=$ $z+c_{2} z^{2}+\ldots \in \mathfrak{A}$ which satisfy the condition $\left\|G_{f}\right\| \leq k$. It follows from the definition that $\mathcal{S}\left(k_{1}\right) \subset \mathcal{S}\left(k_{2}\right)$ if $0 \leq k_{1}<k_{2} \leq 1$. We denote by $\mathcal{S}_{Q}$, $Q \geq 1$, the class of functions $f(z)=z+c_{2} z^{2}+\ldots \in \mathfrak{A}$ which have a 
$Q$-quasiconformal extension onto the whole sphere $\overline{\mathbb{C}}=\mathbb{C} \cup\{\infty\}$. Some geometric considerations imply that $\left\|G_{f}\right\| \leq(Q-1) /(Q+1)$ for $f \in \mathcal{S}_{Q}$ (for details and references see e.g. [13, Ch. 9], [10, Part 2, Ch. 2]). Therefore $\mathcal{S}_{Q} \subset \mathcal{S}(k)$ if $Q \leq(1+k) /(1-k), k<1$. However, for each $k \in(0,1)$ the class $\mathcal{S}(k)$ contains not only $Q$-quasiconformally extendable functions with some $Q \leq(1+k) /(1-k)$. The first example to show that was given by Kühnau in 1971 (see e.g. [10]).

Nevertheless, each function in $\mathcal{S}(k)$ for $k<1$ has a quasiconformal extension onto the whole $\overline{\mathbb{C}}$. This was actually proved by $\mathrm{Ch}$. Pommerenke [13, Ch. 9], who considered the class of meromorphic functions univalent in $\overline{\mathbb{C}} \backslash \bar{E}$ and satisfying the strengthened Grunsky inequality (see [13]). The class $\mathcal{S}=\mathcal{S}(1)$ is the main object of study in the theory of univalent functions. The Koebe function $\mathbf{k}_{1}(z)$ is here of particular importance. This is connected with the fact that the function $\mathbf{k}_{1}(z)$ (as well as its rotations $\left.\mathbf{k}_{\kappa}(z)=\kappa \mathbf{k}_{1}(\bar{\kappa} z),|\kappa|=1\right)$ is extremal for a whole class of traditional functionals on the class $\mathcal{S}$.

So, it is known (see e.g. [12, Ch. 1]) that for any $f \in \mathcal{S}$ and for each $r \in(0,1)$ we have

$$
\begin{aligned}
|f(z)| & \leq r(1-r)^{-2}, \\
\left|z f^{\prime}(z) / f(z)\right| & \leq(1+r) /(1-r),
\end{aligned}
$$

where $|z| \leq r$. Also [2], if $f(z)=z+c_{2} z^{2}+\ldots \in \mathcal{S}$ then for each $n=2,3, \ldots$ we have

$$
\left|c_{n}\right| \leq n
$$

Equality holds in (9), (10) and (11) only for the functions $\mathbf{k}_{\kappa}(z),|\kappa|=1$.

There is a convenient way to separate the functions in $\mathcal{S}$ which are close to the functions $\mathbf{k}_{\kappa}(z),|\kappa|=1$, in the sense of the growth order of Taylor coefficients as $n \rightarrow \infty$, of the growth of their modulus as $r \rightarrow 1$, etc. Namely, W. K. Hayman (see e.g. [12, Ch. 3]) established that for each $f \in \mathcal{S}$ there exists $\alpha(f)=\lim _{r \rightarrow 1-0} M(r, f)(1-r)^{2} \leq 1$, where $M(r, f)=\max _{|z|=r}|f(z)|, r \in(0,1)$. Hayman's index $\alpha(f)$ is equal to 1 only for $f(z)=\mathbf{k}_{\kappa}(z),|\kappa|=1$. For any $f$ with $\alpha(f) \neq 0$ there exists a unique direction of largest growth of $f$, i.e. there exists a unique $\theta(f) \in[0,2 \pi)$ such that $\lim _{r \rightarrow 1-0}\left|f\left(r e^{i \theta(f)}\right)\right|(1-r)^{2}=\alpha(f)$.

For all other functions in $\mathcal{S}$ (i.e. those for which $\alpha(f)=0$ ) the last limit, of course, exists, and is equal to 0 for any value of the argument. In terms of Hayman's index and the direction of largest growth one can estimate the closeness to $\mathbf{k}_{\kappa}$ of each function $f \in \mathcal{S}$ with $\alpha(f) \neq 0$ and $\theta(f)=\arg \kappa,|\kappa|=1$. Rather important results here (see e.g. [12, Ch. 3]) are the asymptotics of the coefficients of univalent functions established first by W. K. Hayman and the Bazilevich inequality for the logarithmic 
coefficients defined for $f \in \mathcal{S}$ by the expansion

$$
\log [f(z) / z]=2 \sum_{n=1}^{\infty} \gamma_{n} z^{n} .
$$

The Milin inequality for the logarithmic coefficients $\gamma_{n}$ (ibid.) is even more important for applications. Though it is not sharp, it does not depend on $\alpha(f)$ and $\theta(f)$ and provides the true logarithmic order of growth for $\mathbf{k}_{\kappa}$, $|\kappa|=1$, for some means. In this section we show that in each class $\mathcal{S}(k)$ $(0 \leq k<1)$ deep analogues of the three above-mentioned and other extremal properties of the function $\mathbf{k}_{\kappa}$ hold, which are realized by the function $f_{k}(z)=$ $z(1-z)^{-1-k} \in \mathcal{S}(k)$ and its rotations (see $[6 ; 7]$ ).

Thereby, a stratification of the analytical properties of univalent functions depending on the norm of the Grunsky operator comes out, which is closely connected with homeomorphic extension and other geometric characteristics. The results of W. K. Hayman, I. E. Bazilevich and I. M. Milin correspond to the case $k=1$.

Since for each $k$ the role of the function $f_{k}$ for the class $\mathcal{S}(k)$ (see [7]) is similar to the extremal role of the Koebe function for the whole class $\mathcal{S}$, we mention some properties of $f_{k}$. The function $w=u+i v=f_{k}(z)$ maps $E$ univalently and conformally onto a domain of the $w$-plane starlike with respect to 0 . This immediately follows from the fact that the normalized logarithmic derivative $z f_{k}^{\prime}(z) / f_{k}(z)$ is in the Carathéodory class of functions regular in $E$ with positive real part. Setting $f_{k}(z)=z(1-z)^{-1}\left(1-\bar{z}^{-1}\right)^{-k}$ for $k<1$ and $z \in \overline{\mathbb{C}} \backslash E$ we obtain a $Q$-quasiconformal extension of $f_{k}$ onto the whole $\overline{\mathbb{C}}$ where $Q=(1+k) /(1-k)[6]$, with the modulus of the complex dilatation of this quasiconformal extension being equal to $k$ identically. Indeed, this corresponds to the fact that the function $g(\zeta)=\zeta \cdot \bar{\zeta}^{k}$ is $Q$-quasiconformal in $|1-\zeta| \leq 1$. In fact, $g$ is a homeomorphism since $\left|\arg \zeta_{1}-\arg \zeta_{2}\right|<\pi$ for any two different $\zeta_{1}$ and $\zeta_{2}$ in the above set. In addition, for $\zeta \neq 0$ we have $\left|g_{\bar{\zeta}}^{\prime} / g_{\zeta}^{\prime}\right|=\left|\zeta k \bar{\zeta}^{k-1} / \bar{\zeta}^{k}\right|=k$. Thus, for $k<1$ and $Q=(1+k) /(1-k)$ the function $f_{k}$ is in the class $\mathcal{S}_{Q}$, for which it plays of course the same remarkable role as for a wider class $\mathcal{S}(k)[6 ; 7]$. Hence we have $\left\|G_{f_{k}}\right\| \leq k$. On the other hand, the growth of the Taylor coefficients as $n \rightarrow \infty$ and also the growth of the modulus of $f_{k}$ as $|z| \rightarrow 1$ cannot exceed the growth of the corresponding majorant for the class $\mathcal{S}\left(\left\|G_{f_{k}}\right\|\right.$ ) (see [7], also (20), (23), (24)). Therefore $k \leq\left\|G_{f_{k}}\right\|$ and hence $\left\|G_{f_{k}}\right\|=k$.

Note, moreover, that the domain $f_{k}(E)$ contains the disk $\{w:|w|<$ $\left.2^{-1-k}\right\}$ and its boundary $\partial f_{k}(E)$ lies in the left half-plane and is a quasicircle symmetric about the $u$-axis with $v=\operatorname{Im} w$ diverging to $\pm \infty$ as $u \rightarrow-\infty$ and with an angular point $w_{0}=-2^{-1-k}$. As $k \rightarrow 1, \partial f_{k}(E)$ tends to the ray $\{w: u \leq-1 / 4, v=0\}$ and for $k=0$ it is the straight line $\{w: u=-1 / 2\}$. 
By the definition of the class $\mathcal{S}(k)$, the Grunsky coefficients of a function $f \in \mathcal{S}(k)$ satisfy, for any complex parameters $x_{n}(n=1,2, \ldots)$, the inequality

$$
\left|\sum_{n, l=1}^{\infty} x_{n} x_{l} \alpha_{n, l}\right| \leq k \sum_{n=1}^{\infty} \frac{\left|x_{n}\right|^{2}}{n}
$$

called the strengthened Grunsky inequality. It is clear that for functions $f(z)=z+c_{2} z^{2}+\ldots$ regular in $E$ and for each $k \in[0,1]$ the inequality (13) gives a necessary and sufficient condition for membership in $\mathcal{S}(k)$. The following inequality for $f \in \mathcal{S}(k)$, equivalent to (13) (since the $x_{n}$ are arbitrary) but more convenient for applications, is given in [7]. For any function $q(w) \not \equiv$ const regular in $\overline{\mathbb{C}} \backslash f(E)$ (the set of such functions $q$ for $f \in \mathcal{S}$ is denoted by $\mathcal{N}(f))$ we have

$$
\sum_{n=1}^{\infty} n\left|a_{n}\right|^{2} \leq k^{2} \sum_{n=1}^{\infty} n\left|a_{-n}\right|^{2},
$$

where the coefficients $a_{n}(n= \pm 1, \pm 2, \ldots)$ are defined by the expansion $q \circ f(z)=\sum_{n=-\infty}^{\infty} a_{n} z^{n}$ which holds in some annulus $\varrho<|z|<1$.

The result of [7] on the equivalence of the two inequalities means that for any $f \in \mathcal{S}$ the representation $\left\|G_{f}\right\|=\sup _{q \in \mathcal{N}(f)}\|f\|_{q}$ holds, where

$$
\|f\|_{q}=\left[\sum_{n=1}^{\infty} n\left|a_{n}\right|^{2} / \sum_{n=1}^{\infty} n\left|a_{-n}\right|^{2}\right]^{1 / 2} .
$$

Hence $\left\|G_{f}\right\|$ is invariant under admissible Möbius transformations (including automorphisms of the disk E). In fact, Ch. Pommerenke [13, Ch. 9], established this property using the strengthened Goluzin inequality for any finite set of points in $E$.

It also follows from the representation of the norm of the Grunsky operator (taking into account that $w^{-1} \in \mathcal{N}(f)$ for any $f \in \mathcal{S}$ ) that if $\left\|G_{f}\right\|=0$ then $f$ is a Möbius transformation. The converse easily follows from (13).

In other words, $\mathcal{S}(0)=\{z /(1-\zeta z): \zeta \in \bar{E}\}$. Recall that in [7] the inequality (14) was used for the definition of the class $\mathcal{S}(k)$. It was actually shown there that this inequality holds for any function in $\mathcal{S}$ for which there exists a regularly measurable extension onto $\overline{\mathbb{C}} \backslash E$ with homeomorphism coefficient not exceeding $k$ and with the function $q$ admissible for this extension [7].

Therefore the class $\mathcal{S}(k)$ contains all those functions in $\mathcal{S}$ for which there exists an entirely regularly measurable extension onto $\overline{\mathbb{C}} \backslash E$ with homeomorphism coefficient at most $k$ (for details see $[7, \S \S 2,4]$ ). 
Now let

$$
q(w)=\sum_{l=1}^{N} x_{l} \log \left[w^{-1}-f^{-1}\left(z_{l}\right)\right]
$$

where the $z_{l}$ are arbitrary points in $E$, and the $x_{l}$ are any complex numbers $(l=1, \ldots, N ; N=1,2, \ldots)$. For such choice of $q(w)$ and for $f \in \mathcal{S}(k)$ the inequality (14) can be rewritten as

$$
\begin{aligned}
\sum_{n=1}^{\infty} n & \left|\sum_{l=1}^{N} x_{l}\left[A_{n}\left(z_{l}\right)+k \frac{\bar{\zeta}_{l}^{n}}{n}\right]\right|^{2} \\
\leq k \sum_{n, l=1}^{N} x_{n} \bar{x}_{l} \log \left[\left(\frac{f^{-1}\left(z_{n}\right)-f^{-1}\left(\zeta_{l}\right)}{z_{n}^{-1}-\zeta_{l}^{-1}}\right) \overline{\left(\frac{f^{-1}\left(z_{l}\right)-f^{-1}\left(\zeta_{n}\right)}{z_{l}^{-1}-\zeta_{n}^{-1}}\right)}\right. & \left.\times \frac{1}{\left(1-z_{n} \bar{z}_{l}\right)^{k}\left(1-\bar{\zeta}_{n} \zeta_{l}\right)^{k}}\right]
\end{aligned}
$$

where

$$
A_{n}(z)=\sum_{l=1}^{\infty} \alpha_{n, l} z^{l} \quad(z \in E),
$$

$\alpha_{n, l}(n, l=1,2, \ldots)$ are the Grunsky coefficients of $f$ and $\zeta_{l}(l=1, \ldots, N)$ are arbitrary points in $E$ (cf. [7]). Even simple individual cases and corollaries of the multiparametric inequality (15) allow one to obtain a fairly exact and visual picture of the growth of some important functionals for the classes $\mathcal{S}(k)$ [7]. That is of particular interest as the sharp estimates for most functionals depending on $k$ must be complicated and therefore unsuitable for applications (see in [7] the same argument for the classes $\mathcal{S}_{Q}$ ). In particular, we have two equivalent inequalities for $f \in \mathcal{S}(k)$ :

$$
\begin{gathered}
\sum_{n=1}^{\infty} n\left|A_{n}(z)\right|^{2} \leq k^{2} \log \frac{1}{1-r^{2}}, \quad|z|=r<1 \\
\sum_{n=1}^{\infty} n\left|A_{n}(z)+k \frac{\bar{\zeta}^{n}}{n}\right|^{2} \\
\leq k \log \left[\left|\frac{f^{-1}(z)-f^{-1}(\zeta)}{z^{-1}-\zeta^{-1}}\right|^{2} \frac{1}{\left(1-|z|^{2}\right)^{k}\left(1-|\zeta|^{2}\right)^{k}}\right]
\end{gathered}
$$

where $z, \zeta \in E$ and $A_{n}(z)(n=1,2, \ldots)$ are defined by $(16)$.

In [5] for any $k \in[0,1]$ the estimates of the logarithmic means and Taylor coefficients for functions in $\mathcal{S}$ satisfying the inequality (17) are proved. Therefore, as was mentioned in [7], those estimates and their corollaries hold for all functions in $\mathcal{S}(k)$. Since the results in [5] are formulated only for the class $\mathcal{S}_{Q}$ for $Q=(1+k) /(1-k)$ let us give the corresponding theorem for 
the class $\mathcal{S}(k)$. Its proof is similar to that of [5], except for the use of Milin's constant [12, Ch. 3]

$$
\delta=\frac{1}{2}\left[\sum_{n=1}^{\infty} \frac{(\log 2)^{n}}{n ! n}-\log \log 2-c\right]=0.3118 \ldots,
$$

where $c$ is the Euler constant.

Theorem 2. Let $f(z)=z+c_{2} z^{2}+\ldots \in \mathcal{S}(k), k \in[0,1]$. Then for $n=1,2, \ldots$ we have the inequalities

$$
\begin{gathered}
\left|c_{n}\right| \leq(a / k)^{k / 2}\left\{f_{k}\right\}_{n}<b n^{k}, \\
|| c_{n+1}|-| c_{n}||<4 n^{\left(k^{2}-1\right) / 2}, \\
\sum_{l=1}^{n} l\left|\gamma_{l}\right|^{2} \leq\left(\frac{1+k}{2}\right)^{2} \sum_{l=1}^{n} \frac{1}{l}+\frac{k(1+k)}{4} \log \frac{e^{2 \delta}}{k},
\end{gathered}
$$

where $a, b$ are absolute constants with $a<1.87, b<1.6 ; \delta$ is defined by (19); $\gamma_{n}(n=1,2, \ldots)$ are the logarithmic coefficients from (12).

For $f_{k}(z)$ we have

$$
\begin{aligned}
& \left\{f_{k}\right\}_{n} \sim n^{k} / \Gamma(1+k) \quad \text { as } n \rightarrow \infty, \\
& \left\{\log \left(f_{k}(z) / z\right)\right\}_{n}=(1+k) / n \quad(n=1,2, \ldots) .
\end{aligned}
$$

Therefore (20) and (22) give the sharp order in $n$ as $n \rightarrow \infty$. For $k=0$, (22) and the first inequality in (20) are sharp, with equality holding if and only if $f(z)=\kappa f_{0}(\bar{\kappa} z),|\kappa|=1$. For $k=1$, (22) coincides with the abovementioned Milin inequality [12] for logarithmic means. As to the inequality (21), except for the case $k=1$ (and, of course, $k=0$ ) the question of the growth order of its left side as $n \rightarrow \infty$ is not yet solved. Recall that sharp estimates (in the sense of the growth order as $|z| \rightarrow 1$ ) of $|f(z)|$ and $\left|f^{\prime}(z)\right|$ are simple corollaries of the inequality (20) (see [7]).

For $k \in[0,1]$, let

$$
\begin{aligned}
& \nu_{n}(k)=\sup _{f \in \mathcal{S}(k)}\left|\{f\}_{n}\right| /\left\{f_{k}\right\}_{n} \quad(n=2,3, \ldots), \quad \nu(k)=\sup _{n} \nu_{n}(k), \\
& \eta(k)=\sup _{f \in \mathcal{S}(k), z \in E}|f(z)| / f_{k}(|z|), \quad \widetilde{\eta}(k)=\sup _{f \in \mathcal{S}(k), z \in E}\left|f^{\prime}(z)\right| / f_{k}^{\prime}(|z|) .
\end{aligned}
$$

It is obvious that $\eta(k), \widetilde{\eta}(k) \leq \nu(k)$. For $k=0$ we have $\nu(0)=\eta(0)=$ $\widetilde{\eta}(0)=1$ (see also [7]). For $k=1$ it is known that $\eta(1)=\widetilde{\eta}(1)=1$ (see (9) and $(10)), \nu_{n}(1)=1(n=2,3, \ldots)$ (see (11)) and therefore $\nu(1)=1$. For each $k \in(0,1)$ the values $\eta(k), \widetilde{\eta}(k)$ and $\nu_{n}(k)(n=2,3, \ldots)$ are strictly greater than one. This is shown by the simplest variation of the function $f_{k}$ obtained by considering an automorphism of the disk $E$. Stronger estimates from below for $\eta(k)$ and $\nu_{2}(k)(0<k<1)$ can be obtained from the known results for the classes $\mathcal{S}_{Q}(Q>1)$ (see e.g. [5;7] and the references there). 
From (20) we find [7]

$$
\eta(k), \widetilde{\eta}(k) \leq \nu(k) \leq(a / k)^{k / 2}<2^{1 / 2} .
$$

These estimates are useful for applications though they are certainly not sharp for $k$ close to 1 .

A strengthened version of the well-known Goluzin theorem on distortion of chords for $f \in \mathcal{S}(k)$ results from the obvious non-negativity of the right side of (18) for $|z|=|\zeta|=r<1$ :

$$
\left|\frac{f(z)-f(\zeta)}{z-\zeta}\right| \geq|f(z) f(\zeta)| r^{-2}\left(1-r^{2}\right)^{k}
$$

Hence for $z=\zeta$, on taking into account (10), we find

$$
|f(z)| \leq 2^{1-k} f_{k}(r), \quad|z| \leq r<1 .
$$

This inequality, as well as the more subtle inequalities (20) and (22) (see also $(23))$, is best possible in the sense of the growth order. For $k$ close to $1,(24)$ gives a more precise estimate of $\eta(k)$ than in $(23)$. Thus, for $\eta(k)$ we have an estimate

$$
\eta(k) \leq \min \left\{(a / k)^{k / 2}, 2^{1-k}\right\}<7 / 5 \quad(a<1.87),
$$

sharp for $k=0$ and 1. A better estimate of $\nu_{n}(k)(n=2,3, \ldots)$ (than the one obtained from (20)) follows from the non-negativity of the right side in (15). Exactly in that manner (by following Fitzgerald's approach for the class $\mathcal{S}$ ) the inequality

$$
\left|\sum_{n, l=1}^{N} Y_{n, l} c_{n} \bar{c}_{l}\right|^{2} \leq \sum_{n, l, \tilde{n}, \tilde{l}=1}^{N} Y_{n, l} \bar{Y}_{\tilde{n}, \tilde{l}} b_{n, \tilde{n}, l, \tilde{l}}
$$

was obtained in [7] for $f(z)=z+c_{2} z^{2}+\ldots \in \mathcal{S}(k), k \in[0,1]$ and $N=$ $1,2, \ldots$, where $Y_{n, l}$ are arbitrary complex numbers, and

$$
b_{n, \tilde{n}, l, \tilde{l}}=\sum_{m=1}^{\min (n, \tilde{n})} \sum_{\tilde{m}=1}^{\min (l, \tilde{l})} c_{n+\tilde{l}+1-m-\tilde{m}} \bar{c}_{\tilde{n}+l+1-m-\tilde{m}}\left\{f_{k}\right\}_{m}\left\{f_{k}\right\}_{\tilde{m}}
$$

for $n, l, \widetilde{n}, \widetilde{l}=1, \ldots, N$. In particular,

$$
\left|c_{N}\right|^{4} \leq \sum_{m, \tilde{m}=1}^{N}\left|c_{2 N+1-m-\tilde{m}}\right|^{2}\left\{f_{k}\right\}_{m}\left\{f_{k}\right\}_{\tilde{m}} .
$$

Calculations on this ground for various $N$ showed that for $k \in(0,1)$ the functions $\nu_{N}(k)$ have a one-modal majorant $\nu(k)$ which does not exceed $7 / 6$. The problem of obtaining majorants for $\nu_{N}(k)(N=2,3, \ldots)$ tending to 1 as $k \rightarrow 1$ remains open. Nevertheless, the above-mentioned ideas and some other ones lead to the conjecture that $\nu(k)(\nu(0)=\nu(1)=1)$ is a 
continuous one-modal function in $[0,1]$ with maximum (within $1.04 \div 1.08$ ) at some point $k_{0} \approx 1 / 2$. It is clear that any improvement of the estimate for $\nu(k)$ allows one, e.g. by using

$$
\eta(k) \leq \min \left\{\nu(k), 2^{1-k}\right\},
$$

to strengthen the estimate for the modulus function for the class $\mathcal{S}(k)$.

Theorem 3. Let $f \in \mathcal{S}(k)(0 \leq k<1)$ and suppose

$$
\alpha_{k}(f)=\limsup _{r \rightarrow 1-0} M(r, f)(1-r)^{1+k}>0 .
$$

Then

$$
\sum_{n=1}^{\infty} n\left|\gamma_{n}-\frac{1+k}{2 n} e^{-i \theta_{0} n}\right|^{2} \leq \frac{k}{2} \log \frac{2^{1-k}}{\alpha_{k}(f)}
$$

where $\theta_{0} \in[0,2 \pi)$ is defined by the condition $f\left(e^{i \theta_{0}}\right)=\infty$ and $\gamma_{n}(n=$ $1,2, \ldots)$ are the logarithmic coefficients from (12).

Proof. From (18) for $\zeta=z$ we have

$$
\sum_{n=1}^{\infty} n\left|A_{n}(z)+k \frac{\bar{z}^{n}}{n}\right|^{2} \leq k \log \left[\left|z^{2} f^{\prime}(z) / f^{2}(z)\right|^{2}\left(1-|z|^{2}\right)^{-2 k}\right], \quad z \in E,
$$

which together with (10) implies, for any $N=1,2, \ldots$,

$$
\sum_{n=1}^{N} n\left|A_{n}(z)+k \frac{\bar{z}^{n}}{n}\right|^{2} \leq 2 k \log \frac{(1+r)^{1-k} r}{(1-r)^{1+k}|f(z)|} .
$$

For each $r \in(0,1)$ let $z_{r}=r e^{i \theta(r)}$ be defined by the condition $M(r, f)=$ $\left|f\left(z_{r}\right)\right|$ and let $\theta_{0}$ be a limit point of $\theta(r)$ as $r \rightarrow 1$. Then, according to (25), $f\left(r e^{i \theta_{0}}\right) \rightarrow \infty$ as $r \rightarrow 1$. Since $k<1, f$ has a quasiconformal extension onto the whole $\overline{\mathbb{C}}$ with a unique pole at $z=e^{i \theta_{0}}$.

Choose a sequence $r_{l} \in(0,1)(l=1,2, \ldots)$ such that $r_{l} \rightarrow 1$ and $\left|f\left(z_{r_{l}}\right)\right|\left(1-r_{l}\right)^{1+k} \rightarrow \alpha_{k}(f)$ as $l \rightarrow \infty$. From (16) and (12) we have $z_{r_{l}} \rightarrow e^{i \theta_{0}}$ as $l \rightarrow \infty$ and

$$
A_{n}\left(z_{r_{l}}\right) \rightarrow \frac{e^{-i \theta_{0} n}}{n}-2 \gamma_{n} \quad(n=1,2, \ldots) .
$$

Therefore from the last inequality we find

$$
\sum_{n=1}^{N} n\left|2 \gamma_{n}-\frac{1+k}{n} e^{-i \theta_{0} n}\right|^{2} \leq 2 k \log \frac{2^{1-k}}{\alpha_{k}(f)} .
$$

Letting $N \rightarrow \infty$ completes the proof.

Remark. For $k=1$ we have $\alpha_{k}(f)=\alpha(f)$, and $\theta_{0}=\theta(f)$ defines the direction of largest growth of $f$; that is why (26) coincides with the 
Bazilevich inequality (see e.g. [12, Ch. 3]). Thus, (26) can be used for all $k \in[0,1]$. The same is true for Theorems 5 and 6 .

Lemma 2 (H. Prawitz, see e.g. [3, Ch. 2]). Let $f \in \mathcal{S}$. Then for any $r \in(0,1)$ and $\lambda>0$,

$$
I_{\lambda}(r)=\frac{1}{2 \pi} \int_{0}^{2 \pi}\left|f\left(r e^{i \theta}\right)\right|^{\lambda} d \theta \leq \lambda \int_{0}^{r} M(\varrho, f)^{\lambda} \frac{d \varrho}{\varrho} .
$$

Lemma 3. For $f \in \mathcal{S}$ and $\mu>0$ let

$$
\beta_{\mu}=\limsup _{r \rightarrow 1-0} M(r, f)(1-r)^{\mu}<\infty .
$$

Then for any $\lambda>\mu^{-1}$,

$$
\limsup _{r \rightarrow 1-0}(1-r)^{\lambda \mu-1} I_{\lambda}(r) \leq \frac{\lambda}{\lambda \mu-1} \beta_{\mu}^{\lambda} .
$$

Proof. For any $\varepsilon>0$ there exists $\Delta \in(0,1)$ such that

$$
M(r, f)(1-r)^{\mu}<\beta_{\mu}+\varepsilon
$$

for all $r \in(1-\Delta, 1)$. From Lemma 2 for such $r$ we have

$$
I_{\lambda}(r) \leq \lambda\left(\int_{0}^{1-\Delta} M(\varrho, f)^{\lambda} \frac{d \varrho}{\varrho}+\frac{1}{1-\Delta} \int_{1-\Delta}^{r} \frac{\left(\beta_{\mu}+\varepsilon\right)^{\lambda}}{(1-\varrho)^{\mu \lambda}} d \varrho\right) .
$$

It follows that

$$
\limsup _{r \rightarrow 1-0}(1-r)^{\mu \lambda-1} I_{\lambda}(r) \leq \frac{\lambda}{(1-\Delta)(\mu \lambda-1)}\left(\beta_{\mu}+\varepsilon\right)^{\lambda},
$$

and it suffices to let $\Delta \rightarrow 0$ and $\varepsilon \rightarrow 0$.

LEMMA 4. In the notation of Lemma 3,

$$
\limsup _{n \rightarrow \infty} \frac{\left|\left\{[f(z) / z]^{\lambda}\right\}_{n}\right|}{\left\{f_{\lambda \mu-1}(z)\right\}_{n}} \leq \frac{\lambda e \Gamma(\lambda \mu)}{\lambda \mu-1} \beta_{\mu}^{\lambda} .
$$

Proof. By the Cauchy integral formula we have for $n=1,2, \ldots$ and $r \in(0,1)$

$$
\left|\left\{[f(z) / z]^{\lambda}\right\}_{n}\right| \leq \frac{1}{2 \pi r^{n+\lambda}} \int_{0}^{2 \pi}\left|f\left(r e^{i \theta}\right)\right|^{\lambda} d \theta .
$$

Hence

$$
\left|\left\{[f(z) / z]^{\lambda}\right\}_{n}\right|(1-r)^{\lambda \mu-1} \leq \frac{(1-r)^{\lambda \mu-1}}{r^{n+\lambda}} I_{\lambda}(r) .
$$

Setting $r=1-n^{-1}(n=2,3, \ldots)$ and using Lemma 3 we obtain the assertion. 
To obtain the asymptotics of the coefficients of a function in $\mathcal{S}(k)$ we slightly modify Milin's Tauberian theorem (see e.g. [12, Ch. 2]).

Theorem 4. Suppose $\omega(z)=\sum_{n=1}^{\infty} A_{n} z^{n}$ is regular in $E, \sum_{n=1}^{\infty} n\left|A_{n}\right|^{2}$ $<\infty$ and

$$
\sup _{n} \operatorname{Re} \sum_{l=1}^{n} A_{l}<\infty .
$$

Then for $\varphi(z)=\exp \omega(z)$ and any $\mu>1 / 2$ we have

$$
\left\{\varphi(z)(1-z)^{-\mu}\right\}_{n} /\left\{(1-z)^{-\mu}\right\}_{n}-\varphi(r)=o(1) \quad(n \rightarrow \infty)
$$

where $r=r(n) \in(0,1)$ satisfies

$$
\log [n(1-r)]=O(1) \quad(n \rightarrow \infty) .
$$

In [12, Ch.2] a stronger result is proved, but under a more restrictive condition than (27): $\operatorname{Re} \sum_{l=1}^{\infty} A_{l}=O(1)(n \rightarrow \infty)$. For $\mu=1$ and 2 and $r=1-n^{-1}$ the assertion of Theorem 4 with the condition (27) is proved in [3, Ch. 5]. The proof of Theorem 4 in the general case differs slightly from that in [12].

TheOREM 5. Let $f(z)=z+c_{2} z^{2}+\ldots \in \mathcal{S}(k), k \in[0,1]$. Then

$$
\lim _{n \rightarrow \infty}\left[\frac{c_{n}}{\left\{f_{k}\right\}_{n}}-\frac{e^{-i \theta_{0} n} f\left(r e^{i \theta_{0}}\right)}{f_{k}(r)}\right]=0
$$

where $e^{i \theta_{0}}$ is the pole of $f(z)$ (if there is no pole, then $\theta_{0}$ is any real number) and $r$ satisfies the condition (28).

Pr o of. One can assume that $\theta_{0}=0$. First suppose that the condition (25) holds. For $\lambda>0$ let

$$
\omega(z)=\sum_{n=1}^{\infty} A_{n} z^{n}=\log \left[\frac{f(z)}{z}(1-z)^{1+k}\right]^{\lambda} .
$$

Then the assumptions of Theorem 4 are satisfied. Indeed, by (12), from Theorem 3 we have

$$
\sum_{n=1}^{\infty} n\left|A_{n}\right|^{2}=4 \lambda^{2} \sum_{n=1}^{\infty} n\left|\gamma_{n}-\frac{1+k}{2 n}\right|^{2} \leq 2 \lambda^{2} k \log \frac{2^{1-k}}{\alpha_{k}(f)}<\infty .
$$

Moreover,

$$
\operatorname{Re} \sum_{n=1}^{\infty} A_{n} r^{n}=\lambda \log \frac{|f(r)|}{f_{k}(r)}<\infty \quad \text { as } r \rightarrow 1 .
$$

It remains to take into account that

$$
\operatorname{Re} \sum_{l=1}^{n} A_{l}=\operatorname{Re} \sum_{l=1}^{\infty} A_{l} r^{l}+o(1) \quad(n \rightarrow \infty) .
$$


According to Theorem 4 for $\mu=\lambda(1+k)>1 / 2$ and under the condition (28),

$$
\lim _{n \rightarrow \infty}\left(\frac{\left\{[f(z) / z]^{\lambda}\right\}_{n}}{\left\{f_{\mu-1}(z)\right\}_{n+1}} e^{i \theta_{0} n}-\left[\frac{e^{-i \theta_{0}} f\left(r e^{i \theta_{0}}\right)}{f_{k}(r)}\right]^{\lambda}\right)=0 .
$$

If the condition (25) is not satisfied, then $\lim _{r \rightarrow 1-0} M(r, f)(1-r)^{1+k}=0$ and therefore $\lim _{r \rightarrow 1-0}\left[e^{-i \theta_{0}} f\left(r e^{i \theta_{0}}\right) / f_{k}(r)\right]=0$. In view of Lemma 4 we conclude that (29) holds for $\lambda(1+k)>1$ also in this case.

Therefore, if $k+\alpha_{k}(f)>0$, then (29) holds for all $\lambda \geq 1$. Suppose $k=\alpha_{k}(f)=0$. Then $f(z)=z /(1-\zeta z)$, where $\zeta \in E$. Since $\left|c_{n}\right|=|\zeta|^{n} \rightarrow 0$ as $n \rightarrow \infty$, the proof of the theorem is complete.

THEOREM 6. In the notation of Theorem 5, under the assumption (25), for $k>1 / 2$ we have

$$
\lim _{n \rightarrow \infty}\left[\frac{c_{n}-e^{-i \theta_{0}} c_{n-1}}{\left\{f_{k-1}(z)\right\}_{n}}-\frac{e^{-i \theta_{0} n} f\left(e^{i \theta_{0}} r\right)}{f_{k}(r)}\right]=0 .
$$

Proof. As in the proof of Theorem 5, using Theorem 4 we obtain

$$
\lim _{n \rightarrow \infty}\left(\frac{\left\{[f(z) / z]^{\lambda}\left(1-e^{-i \theta_{0}} z\right)\right\}_{n}}{\left\{f_{\mu-1}(z)\right\}_{n+1}} e^{i \theta_{0} n}-\left[\frac{e^{-i \theta_{0}} f\left(r e^{i \theta_{0}}\right)}{f_{k}(r)}\right]^{\lambda}\right)=0
$$

where $\mu=\lambda(1+k)-1>1 / 2$. That is, provided $\lambda(1+k)>3 / 2$ we have

$$
\lim _{n \rightarrow \infty}\left(\frac{\left\{[f(z) / z]^{\lambda}\right\}_{n}-e^{-i \theta_{0}}\left\{[f(z) / z]^{\lambda}\right\}_{n-1}}{\left\{f_{\mu-1}(z)\right\}_{n+1}} e^{i \theta_{0} n}-\left[\frac{e^{-i \theta_{0}} f\left(r e^{i \theta_{0}}\right)}{f_{k}(r)}\right]^{\lambda}\right)=0 \text {. }
$$

Hence for $\lambda=1$ the assertion of the theorem follows.

$\S$ 3. $\tau$-Norm and the classes $\mathfrak{M}^{*}(k)$. Let $\mathfrak{M}^{*}$ be the class of pairs of univalent functions $f(z)$ and $h(z)$ mapping $E$ conformally onto nonoverlapping domains of the $w$-plane. Many problems for the known classes: bounded functions, Bieberbach-Eilenberg functions, Gelfer functions and other classes of functions regular and univalent in $E$ lead to the study of the general properties of the class $\mathfrak{M}^{*}$, which is also of independent interest. In 1961 N. A. Lebedev (see e.g. [11, Ch. 3]) proved the now well-known area theorem for systems of univalent functions without common values, using as a parameter any function with regular and single-valued derivative in the uncovered part of the plane. His theorem can be applied in proving many important results for the class $\mathfrak{M}^{*}$. Using those geometric ideas he and the other authors obtained a number of estimates both for pairs of functions without common values and for functions of the above-mentioned classes. Sometimes a strengthening of Lebedev's theorem for pairs of functions $\{f, h\}$ in $\mathfrak{M}^{*}$ is possible, e.g. an information on the quantitative characteristic of their homeomorphic assembling leads to more subtle estimates [6]. In this section we consider some properties of pairs of functions in $\mathfrak{M}^{*}$ depending 
on the values of some functional which appears as a natural generalization of the norm of the Grunsky operator, and introduce the corresponding classes of pairs of functions.

For a pair $\{f, h\} \in \mathfrak{M}^{*}$ denote by $\mathcal{M}(f, h)$ the set of functions $q(w) \not \equiv$ const, having in $\mathbb{C} \backslash(f(E) \cup h(E))$ a regular and single-valued derivative. For $q \in \mathcal{M}(f, h)$ denote by $\mathcal{T}_{q}(f, h)[6]$ the class of orientation preserving homeomorphisms $F(z)$ of the extended complex plane onto itself, each of which is conformal outside some annulus $d_{F}=\{z: 0<r(F) \leq|z| \leq$ $R(F)<\infty\}$ and satisfies the following conditions:

1) for all $z \in E$ and some $\theta_{1}(F), \theta_{2}(F) \in[0,2 \pi)$

$$
f(z)=F\left(z r e^{i \theta_{1}}\right), \quad h(z)=F\left(R e^{i \theta_{2}} / z\right) ;
$$

2) in the annulus $d_{F}, F$ is a regularly measurable mapping with the admissible function $q[7]$.

The coefficient of homeomorphic q-assembling of $f$ and $h$ is defined by

$$
K_{q}(f, h)=\inf _{F \in \mathcal{T}_{q}(f, h)} \omega(q \circ F)
$$

where

$$
\omega(u)=\left[\iint_{d_{F}}\left|u_{\bar{\zeta}}^{\prime}\right|^{2} d \sigma / \iint_{d_{F}}\left|u_{\zeta}^{\prime}\right|^{2} d \sigma\right]^{1 / 2}, \quad u=q \circ F,
$$

for $\mathbf{D}(u) \neq 0$ and $\omega(u)=0$ otherwise (cf. [6]). Here and below $\mathbf{D}(u)$ is the generalized Dirichlet integral [14]

$$
\iint_{d_{F}}\left(\left|u_{\zeta}^{\prime}\right|^{2}+\left|u_{\bar{\zeta}}^{\prime}\right|^{2}\right) d \sigma
$$

If $\mathcal{T}_{q}(f, h)$ is empty then let $K_{q}(f, h)=1$.

The coefficient of homeomorphic assembling of $f$ and $h$ is defined as $K(f, h)=\sup K_{q}(f, h)$ where the supremum is taken over all functions $q \in \mathcal{M}(f, h)$ for which $\mathcal{T}_{q}(f, h) \neq \emptyset$, if such functions exist; otherwise we set $K(f, h)=1$. From the definition it follows that $0 \leq K_{q}(f, h) \leq K(f, h) \leq 1$.

Let $q \in \mathcal{M}(f, h),\{f, h\} \in \mathfrak{M}^{*}$. Then in some annulus $\varrho<|z|<1$ we have the expansions

(30) $q \circ f(z)=\sum_{n=-\infty}^{\infty} a_{n} z^{n}+\beta \log z, \quad q \circ h(z)=\sum_{n=-\infty}^{\infty} b_{n} z^{n}-\beta \log z$.

Define

$$
\begin{aligned}
& S_{q}(f, h)=\sum_{n=1}^{\infty} n\left(\left|a_{-n}\right|^{2}-\left|b_{n}\right|^{2}+\left|b_{-n}\right|^{2}-\left|a_{n}\right|^{2}\right)+2 \operatorname{Re} \bar{\beta}\left(b_{0}-a_{0}\right), \\
& P_{q}(f, h)=\sum_{n=1}^{\infty} n\left(\left.|| a_{-n}\right|^{2}-\left|b_{n}\right|^{2}|+|\left|b_{-n}\right|^{2}-\left|a_{n}\right|^{2} \mid\right)+2\left|\operatorname{Re} \bar{\beta}\left(b_{0}-a_{0}\right)\right| .
\end{aligned}
$$


Since the area of the image of the set $\overline{\mathbb{C}} \backslash(f(E) \cup h(E))$ under a fixed branch of the function $q(w)$ is equal to $\pi S_{q}[11$, Ch. $3 ; 6]$, we have $0 \leq S_{q} \leq P_{q}$.

Now define

$$
\tau_{q}(f, h)=\left[\left(P_{q}-S_{q}\right) /\left(P_{q}+S_{q}\right)\right]^{1 / 2} \quad \text { if } P_{q} \neq 0 ;
$$

otherwise set $\tau_{q}(f, h)=1$ if $\mathcal{T}_{q}(f, h)=\emptyset$ and $\tau_{q}(f, h)=0$ if $\mathcal{T}_{q}(f, h) \neq \emptyset$.

We define the $\tau$-norm on $\mathfrak{M}^{*}$ by

$$
\tau(f, h)=\sup _{q \in \mathcal{M}(f, h)} \tau_{q}(f, h) .
$$

From (31) it follows that $0 \leq \tau_{q}(f, h) \leq \tau(f, h) \leq 1$ for all $\{f, h\} \in \mathfrak{M}^{*}$ and $q \in \mathcal{M}(f, h)$.

Since $\tau_{q \circ l^{-1}}(l \circ f, l \circ h)=\tau_{q}(f, h)$ for any Möbius transformation $l(w)$, it is clear that the $\tau$-norm (as well as the coefficient of homeomorphic assembling [6]) is invariant under Möbius transformations of the $w$-plane.

If $\tau(f, h)=0$ for a pair $\{f, h\} \in \mathfrak{M}^{*}$ then $f$ and $h$ are Möbius transformations satisfying $h(z)=f(1 /(\zeta z))$ for all $z \in E$ where $\zeta \in \bar{E} \backslash\{0\}$. Indeed, let $l$ be a Möbius transformation such that $l \circ f(0)=0, l \circ h(0)=\infty$. Then $q=\log \circ l \in \mathcal{M}(f, h)$ and $\tau_{q}(f, h)=0$. We have

$$
\begin{aligned}
q \circ f(z) & =\sum_{n=0}^{\infty} a_{n} z^{n}+\log z, \quad q \circ h(z)=\sum_{n=0}^{\infty} b_{n} z^{n}-\log z, \\
S_{q} & =2 \operatorname{Re}\left(b_{0}-a_{0}\right)-\sum_{n=1}^{\infty} n\left(\left|a_{n}\right|^{2}+\left|b_{n}\right|^{2}\right) \geq 0, \\
P_{q} & =2 \operatorname{Re}\left(b_{0}-a_{0}\right)+\sum_{n=1}^{\infty} n\left(\left|a_{n}\right|^{2}+\left|b_{n}\right|^{2}\right)=S_{q},
\end{aligned}
$$

and our assertion follows. The converse easily follows from expansions (30).

We now show that the $\tau$-norm functional is an extension of the Grunsky operator norm to the class $\mathfrak{M}^{*}$. Assume first that for $f \in \mathcal{S}$ the set $B=$ $\overline{\mathbb{C}} \backslash f(E)$ has interior points. Then the quantities $\|f\|_{q}$ for $q \in \mathcal{N}(f)$ and $\left\|G_{f}\right\|$ (see $\S 2)$ are obviously limiting values of $\tau_{q}(f, h)$ and $\sup _{q \in \mathcal{N}(f)} \tau_{q}(f, h) \leq$ $\tau(f, h)$, resp., for a sequence of pairs $\{f, h\} \in \mathfrak{M}^{*}$ such that the image domain $h(E)$ shrinks to the point $h(0) \in \stackrel{\circ}{B}$. In the general case let

$$
f_{\varrho}(z)=f(\varrho z), \quad \varrho \in(0,1) .
$$

Then

$$
\lim _{\varrho \rightarrow 1-0}\left\|f_{\varrho}\right\|_{q}=\|f\|_{q}, \quad \lim _{\varrho \rightarrow 1-0}\left\|G_{f_{\varrho}}\right\|=\left\|G_{f}\right\| .
$$

It is clear that the set $\overline{\mathbb{C}} \backslash f_{\varrho}(E)$ has interior points. Thus, Grunsky operator norm can be in any case considered as a limiting case of $\tau$-norm for the class of pairs $\mathfrak{M}^{*}$ on $\mathcal{S}$. For the $\tau$-norm we have an important analogue of the 
interconnection between the value of $\left\|G_{f}\right\|$ and homeomorphic extension of $f \in \mathcal{S}$ (see $\S 2$ and $[6 ; 7]$ ). We formulate it in the following theorem whose assertion differs a little from that of Theorem 2 in [6].

Theorem 7. Let $\{f, h\} \in \mathfrak{M}^{*}$ and $q \in \mathcal{M}(f, h)$. Then

$$
\tau_{q}(f, h) \leq K_{q}(f, h) .
$$

If $\mathcal{T}_{q}(f, h)=\emptyset$, then equality holds in $(32)$ if and only if $\sigma[q(\mathbb{C} \backslash(f(E) \cup$ $h(E)))]=0$. If $\mathcal{T}_{q}(f, h) \neq \emptyset$, then equality holds in (32) if and only if

$$
\inf _{F \in \mathcal{T}_{q}(f, h)} \mathbf{D}(q \circ F)=P_{q}(f, h)
$$

and there exist real $x \geq 1$ and $\alpha$ such that the coefficients $\beta$ and $a_{n}, b_{n}$ defined by (30) satisfy

$$
\begin{aligned}
& \left|\operatorname{Re} \bar{\beta}\left(b_{0}-a_{0}\right)\right|=|\beta|^{2} \log x, \quad\left|b_{0}-a_{0}-i \alpha \beta\right|=|\beta| \log x, \\
& \left(x^{n}+x^{-n}\right) a_{n} \bar{b}_{-n} e^{i \alpha n}=\left|a_{n}\right|^{2}+\left|b_{-n}\right|^{2} \quad(n= \pm 1, \pm 2, \ldots) .
\end{aligned}
$$

Moreover, for any $F \in \mathcal{T}_{q}(f, h)$ with $d_{F}=\{\zeta: r(F) \leq|\zeta| \leq R(F)\}$, we have

$$
\mathbf{D}(q \circ F) \geq P_{q}(f, h),
$$

with equality holding if and only if for some real $\alpha$ and $x=R / r$ the conditions (33) hold and if either $x \neq 1$ and

$$
\begin{aligned}
q \circ F(\zeta)= & \sum_{n=1}^{\infty}\left[a_{n}\left(\frac{r}{\overline{\zeta e^{i \alpha}}}\right)^{n}+a_{-n}\left(\frac{r}{\zeta e^{i \alpha}}\right)^{n}\right] \frac{R^{2 n}-|\zeta|^{2 n}}{R^{2 n}-r^{2 n}} \\
& +\sum_{n=1}^{\infty}\left[b_{n}\left(\frac{R}{\zeta}\right)^{n}+b_{-n}\left(\frac{R}{\bar{\zeta}}\right)^{n}\right] \frac{|\zeta|^{2 n}-r^{2 n}}{R^{2 n}-r^{2 n}} \\
& +\left[\left(a_{0}+i \alpha \beta\right) \log \frac{R}{|\zeta|}+b_{0} \log \frac{|\zeta|}{r}\right] / \log \frac{R}{r}+\beta i \arg \zeta \quad\left(\zeta \in d_{F}\right),
\end{aligned}
$$

or $x=1$ and $F(\zeta), f(z)$ and $h(z)=f\left(e^{i \alpha} / z\right)$ are Möbius transformations.

The proof of Theorem 7 is a slight modification of that of Theorem 2 in $[6]$.

Corollary. Let $\{f, h\} \in \mathfrak{M}^{*}$ and suppose that $\bigcap_{q \in \mathcal{M}(f, h)} \mathcal{T}_{q}(f, h) \neq$ $\emptyset$, that is, $f$ and $h$ can be assembled by an entirely regularly measurable homeomorphism. Then $\tau(f, h) \leq K(f, h)$. Moreover, if $f$ and $h$ can be assembled by a Q-quasiconformal homeomorphism, then $K(f, h) \leq(Q-$ $1) /(Q+1)$.

Remark. If in the situation of Theorem 7 we suppose that $f \in \mathcal{S}$, $q \in \mathcal{N}(f)$ and the image domain $h(E)$ shrinks to $h(0)$ then we obtain as a limiting case of this theorem a statement which differs slightly from Theorem 6 in [6] which is a limiting version of Theorem 2 (ibid.). 
Denote by $\mathfrak{M}^{*}(k), k \in[0,1]$, the class of all pairs $\{f, h\}$ in $\mathfrak{M}^{*}$ for which $\tau(f, h) \leq k$. The class $\mathfrak{M}^{*}(1)$ coincides with $\mathfrak{M}^{*}$. It follows from the Corollary of Theorem 7 that $\mathfrak{M}^{*}(k)$ contains all pairs $\{f, h\}$ for which the coefficient of homeomorphic assembling $K(f, h)$ does not exceed $k$. According to D. Aharonov [1], some results for pairs in $\mathfrak{M}^{*}$ without common values can be conveniently formulated in terms of pairs of functions $f, g \in \mathfrak{A}$ such that $f\left(z_{1}\right) g\left(z_{2}\right) \neq 1$ for any $z_{1}, z_{2} \in E$. The class of such pairs is denoted by $\mathfrak{M}_{A}^{*}$. For $\{f, g\} \in \mathfrak{M}_{A}^{*}$ we define $\tau_{q}(f, g), \tau(f, g), K_{q}(f, g)$ and $K(f, g)$ as the corresponding values for $\left\{f, g^{-1}\right\} \in \mathfrak{M}^{*}$ and $q \in \mathcal{M}\left(f, g^{-1}\right)$. We set $\mathfrak{M}_{A}^{*}(k)=\left\{\{f, g\} \in \mathfrak{M}_{A}^{*}:\left\{f, g^{-1}\right\} \in \mathfrak{M}^{*}(k)\right\}, k \in[0,1]$. It follows from the previous considerations that

$$
\mathfrak{M}_{A}^{*}(0)=\left\{\left\{a_{1} z, b_{1} z\right\}: 0<\left|a_{1} b_{1}\right| \leq 1\right\} .
$$

THEOREM 8. Let $\{f, g\} \in \mathfrak{M}_{A}^{*}(k), k \in[0,1]$. Then

$$
\sum_{n=1}^{\infty} n\left(\left|\alpha_{n}\right|^{2}+\left|\beta_{n}\right|^{2}\right) \leq-2 k \log \left|f^{\prime}(0) g^{\prime}(0)\right|
$$

where the coefficients $\alpha_{n}, \beta_{n}$ are defined by the expansions

$$
\log \frac{f(z)}{z}=\sum_{n=0}^{\infty} \alpha_{n} z^{n}, \quad \log \frac{g(z)}{z}=\sum_{n=0}^{\infty} \beta_{n} z^{n},
$$

and equality holds if and only if $\tau_{\log }(f, g)=k$.

The proof follows immediately from the definitions.

Among many corollaries of Theorem 8 (for similar results and references see [6]) we note the following inequalities for any pair $\{f, g\} \in \mathfrak{M}_{A}^{*}(k)$ :

$$
\begin{gathered}
\int_{|z|=1}|f(z)|^{2}|d z| \cdot \int_{|z|=1}|g(z)|^{2}|d z| \leq 4 \pi^{2}\left|f^{\prime}(0) g^{\prime}(0)\right|^{2(1-k)}, \\
\left|\{f\}_{n} \cdot\{g\}_{n}\right|<\frac{e^{-c}}{n}\left|f^{\prime}(0) g^{\prime}(0)\right|^{1-k} \quad(n=2,3, \ldots)
\end{gathered}
$$

where $c$ is the Euler constant.

These inequalities are obtained from Theorem 8 by means of Lemma 1 .

Another transfer of $\tau$-norm on $\mathcal{S}$ is worth mentioning. For $f \in \mathcal{S}$ let $\mathcal{P}(f)[6]$ denote the class of rational functions $t$ of order two for which there exist two single-valued branches $f_{1}$ and $f_{2}$ of the function $t^{-1} \circ f(z)$ in $E$. It is clear that then $\left\{f_{1}, f_{2}\right\} \in \mathfrak{M}^{*}$ and $q^{*}=q \circ t \in \mathcal{M}\left(f_{1}, f_{2}\right)$ if $q \in \mathcal{N}(f)$. We have

$$
q^{*} \circ f_{1}(z)=q^{*} \circ f_{2}(z)=q \circ f(z) .
$$

According to the previous facts the value $\tau_{q}^{*}(f)=\tau_{q^{*}}\left(f_{1}, f_{2}\right)$ is defined by 
(30) and (31) granting that in the considered case

$$
S_{q^{*}}=2 \sum_{n=1}^{\infty} n\left(\left|a_{-n}\right|^{2}-\left|a_{n}\right|^{2}\right), \quad P_{q^{*}}=\left.2 \sum_{n=1}^{\infty} n|| a_{-n}\right|^{2}-\left|a_{n}\right|^{2} \mid .
$$

Now define the functional

$$
\tau^{*}(f)=\sup _{q \in \mathcal{N}(f)} \tau_{q}^{*}(f),
$$

which is the above-mentioned transfer of $\tau$-norm on $\mathcal{S}$. For $f \in \mathcal{S}$ and $q \in \mathcal{N}(f)$ let

$$
K_{q}^{*}(f)=\inf _{t \in \mathcal{P}(f)} K_{q \circ t}\left(f_{1}, f_{2}\right), \quad K^{*}(f)=\sup _{q \in \mathcal{N}(f)} K_{q}^{*}(f)
$$

where the supremum is taken over those functions $q$ for which

$$
\bigcup_{t \in \mathcal{P}(f)} \mathcal{T}_{q \circ t}\left(f_{1}, f_{2}\right) \neq \emptyset
$$

if there are any; otherwise let $K^{*}(f)=1$. The value $K_{q}^{*}(f)$ will be called the coefficient of homeomorphic q-stratification, and $K^{*}(f)$ the coefficient of homeomorphic stratification for $f \in \mathcal{S}$ [6]. Using Theorem 7 one proves the following result, which is a slight modification of Theorem 8 in [6].

Theorem 9. Let $f \in \mathcal{S}$ and $q \in \mathcal{N}(f)$. Then

$$
\tau_{q}^{*}(f) \leq K_{q}^{*}(f) .
$$

For the discussion of all cases of equality cf. Theorem 7 .

Corollary. Let $f \in \mathcal{S}$ and suppose $\bigcap_{q \in \mathcal{N}(f)} \bigcup_{t \in \mathcal{P}(f)} \mathcal{T}_{q \circ t}\left(f_{1}, f_{2}\right) \neq \emptyset$. Then $\tau^{*}(f) \leq K^{*}(f)$.

Two classes of univalent functions: Gelfer's $\mathcal{D}^{*}$ and Bieberbach-Eilenberg's $\mathcal{R}^{*}$ are closely connected with stratification into pairs of functions of the class $\mathcal{S}$. The classes $\mathcal{D}^{*}$ and $\mathcal{R}^{*}$ consist of regular functions univalent in $E$ of the form $\varphi(z)=1+b_{1} z+\ldots$ and $g(z)=c_{1} z+\ldots$ respectively such that for any $z_{1}, z_{2} \in E$ the conditions $\varphi\left(z_{1}\right)+\varphi\left(z_{2}\right) \neq 0$ and $g\left(z_{1}\right) g\left(z_{2}\right) \neq 1$ hold. It is clear that if $\varphi \in \mathcal{D}^{*}$ then $\{\varphi,-\varphi\} \in \mathfrak{M}^{*}$ and if $g \in \mathcal{R}^{*}$ then $\{g, g\} \in \mathfrak{M}_{A}^{*}$. Thus, many properties of univalent Bieberbach-Eilenberg functions are particular cases of properties of pairs in $\mathfrak{M}_{A}^{*}$.

Let $f \in \mathcal{S}$ and suppose $f(z) \neq w$. Then $\varphi(z)=[1-f(z) / w]^{1 / 2}=$ $1-(1 /(2 w)) z+\ldots \in \mathcal{D}^{*}$. The converse assertion is also true: for any $\varphi(z)=1+b_{1} z+\ldots \in \mathcal{D}^{*}$ the function $f(z)=\left[\varphi^{2}(z)-1\right] /\left(2 b_{1}\right) \in \mathcal{S}$ and $f(z) \neq w=-1 /\left(2 b_{1}\right)$. As $t(u)=w\left(1-u^{2}\right) \in \mathcal{P}(f)$ we have $\tau^{*}(f) \leq$ $\tau(\varphi,-\varphi)$. On the other hand, if $\varphi \in \mathcal{D}^{*}$ then $g(z)=(\varphi(z)-1) /(\varphi(z)+$ $1) \in \mathcal{R}^{*}$ and $\tau(\varphi,-\varphi)=\tau(g, g)$. The above-mentioned properties of the $\tau$ norm for the class $\mathcal{D}^{*}$ allow one to study the properties of Gelfer univalent functions depending on the value of this functional. For $k \in[0,1]$ let $\mathcal{D}^{*}(k)$ 
be the subclass of those $\varphi \in \mathcal{D}^{*}$ for which $\{\varphi,-\varphi\} \in \mathfrak{M}^{*}(k)$. In particular, $\mathcal{D}^{*}(1)=\mathcal{D}^{*}$ and $\mathcal{D}^{*}(0)=\{(1+\zeta z) /(1-\zeta z): \zeta \in \bar{E}\}$. Consider the problem of estimating the Taylor coefficients of functions $\varphi(z)=1+b_{1} z+\ldots$ in $\mathcal{D}^{*}(k)$.

Recall Gelfer's result: $\sup _{\mathcal{D}^{*}}\left|b_{1}\right|=2$, and the estimate from $[6]: \sup _{\mathcal{D}^{*}}\left|b_{n}\right|$ $<2.3(n=2,3, \ldots)$ (for details and references, see $[6]$ ).

From this, replacing in the proof of Theorem 10 of [6] the value of $K(\varphi,-\varphi)$ by $\tau(\varphi,-\varphi)$ we obtain the following theorem.

TheOREM 10. Let $\varphi(z)=1+b_{1} z+\ldots \in \mathcal{D}^{*}(k), k \in[0,1]$. Then

$$
\left|b_{1}\right| \leq 2, \quad\left|b_{2}\right| \leq 2+(k / 2)^{4}, \quad\left|b_{n}\right| \leq 2+\min \{k / 2 ; 0.3\} \quad(n \geq 3) .
$$

Equality holds in the first inequality for all $k$, and in the second and third inequalities for $k=0$, if and only if $\varphi(z)=(1+\kappa z) /(1-\kappa z),|\kappa|=1$.

\section{References}

[1] D. Aharonov, A generalization of a theorem of J. A. Jenkins, Math. Z. 110 (1969), $218-222$.

[2] L. de Branges, A proof of the Bieberbach conjecture, Acta Math. 154 (1985), 137-152.

[3] P. L. Duren, Univalent Functions, Springer, Berlin 1983.

[4] A. Z. Grinshpan, An application of the area principle to Bieberbach-Eilenberg functions, Mat. Zametki 11 (1972), 609-618 (in Russian); English transl.: Math. Notes 11 (1972), 371-377.

[5] - On the growth of coefficients of univalent functions with a quasiconformal extension, Sibirsk. Mat. Zh. 23 (2) (1982), 208-211 (in Russian).

[6] - Coefficient inequalities for conformal mappings with homeomorphic extension, Sibirsk. Mat. Zh. 26 (1) (1985), 49-65 (in Russian); English transl.: Siberian Math. J. 26 (1) (1985), 37-50.

[7] -, Univalent functions and regularly measurable mappings, Sibirsk. Mat. Zh. 27 (6) (1986), 50-64 (in Russian); English transl.: Siberian Math. J. 27 (6) (1986), 825-837.

[8] - Method of exponentiation for univalent functions, in: Theory of Functions and Applications (Proc. Conf. Saratov 1988), Part 2, Izdat. Saratov. Univ., Saratov 1990, 72-74 (in Russian).

[9] A. Z. Grinshpan and I. M. Milin, Simply connected domains with finite logarithmic area and Riemann mapping functions, in: Constantin Carathéodory: An International Tribute, World Scientific, Singapore, to appear.

[10] S. L. Krushkal' and R. Kühnau, Quasiconformal Mappings - New Methods and Applications, Nauka, Sibirsk. Otdel., Novosibirsk 1984 (in Russian).

[11] N. A. Lebedev, The Area Principle in the Theory of Univalent Functions, Nauka, Moscow 1975 (in Russian).

[12] I. M. Milin, Univalent Functions and Orthonormal Systems, Nauka, Moscow 1971 (in Russian); English transl.: Amer. Math. Soc., Providence 1977.

[13] Ch. Pommerenke, Univalent Functions, Vandenhoeck und Ruprecht, Göttingen 1975 . 
[14] S. L. Sobolev, Some Applications of Functional Analysis in Mathematical Physics, Izdat. Sibirsk. Otdel. Akad. Nauk SSSR, Novosibirsk 1962 (in Russian).

[15] O. Teichmüller, Untersuchungen über konforme und quasikonforme Abbildungen, Deutsche Math. 3 (6) (1938), 621-678.

NPO CNITA

BUKHARESTSKAYA, 1

LENINGRAD 192102

U.S.S.R.

Reçu par la Rédaction le 20.4.1990 